\title{
Binding modes of decavanadate to myosin and inhibition of the actomyosin ATPase activity
}

\author{
Teresa Tiago $^{\mathrm{a}, \mathrm{b}, \mathrm{d}, *, 1}$, Paulo Martel ${ }^{\mathrm{a}, \mathrm{c}, 1}$, Carlos Gutiérrez-Merino ${ }^{\mathrm{d}}$, Manuel Aureliano ${ }^{\mathrm{a}, \mathrm{b}}$

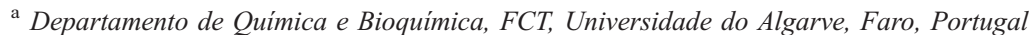 \\ ${ }^{\mathrm{b}}$ CCMar, Universidade do Algarve, Faro, Portugal \\ ${ }^{\mathrm{c}}$ CBME, Universidade do Algarve, Faro, Portugal \\ ${ }^{\mathrm{d}}$ Departamento de Bioquímica y Biología Molecular, Facultad de Ciencias, Universidad de Extremadura, Badajoz, Spain
}

Received 9 November 2006; received in revised form 15 January 2007; accepted 6 February 2007

Available online 20 February 2007

\begin{abstract}
Decavanadate, a vanadate oligomer, is known to interact with myosin and to inhibit the ATPase activity, but the putative binding sites and the mechanism of inhibition are still to be clarified. We have previously proposed that the decavanadate $\left(\mathrm{V}_{10} \mathrm{O}_{28}^{6-}\right)$ inhibition of the actin-stimulated myosin ATPase activity is non-competitive towards both actin and ATP. A likely explanation for these results is that $\mathrm{V}_{10}$ binds to the so-called back-door at the end of the Pi-tube opposite to the nucleotide-binding site. In order to further investigate this possibility, we have carried out molecular docking simulations of the $\mathrm{V}_{10}$ oligomer on three different structures of the myosin motor domain of Dictyostelium discoideum, representing distinct states of the ATPase cycle. The results indicate a clear preference of $\mathrm{V}_{10}$ to bind at the back-door, but only on the "open" structures where there is access to the phosphate binding-loop. It is suggested that $\mathrm{V}_{10}$ acts as a "back-door stop" blocking the closure of the 50$\mathrm{kDa}$ cleft necessary to carry out ATP- $\gamma$-phosphate hydrolysis. This provides a simple explanation to the non-competitive behavior of $\mathrm{V}_{10}$ and spurs the use of the oligomer as a tool to elucidate myosin back-door conformational changes in the process of muscle contraction.
\end{abstract}

(C) 2007 Elsevier B.V. All rights reserved.

Keywords: Back-door; Decavanadate; Myosin; Molecular Docking

\section{Introduction}

Myosin is a highly specialized protein involved in the process of muscle contraction, which along with actin converts the chemical energy of ATP hydrolysis into mechanical work. The motor function of myosin is located on its globular head, the subfragment-1 (S1), which contains both the nucleotide and actin binding sites [1]. The triphosphate portion of ATP is tightly bound in a tube-like structure, termed the Pi-tube, located at the bottom of the active site [2,3]. On the basis of high-resolution Xray structures of the catalytic domain of Dictyostelium discoideum myosin (S1dC) with several nucleotide analogues [4-9], it has been proposed the existence of a secondary

\footnotetext{
* Corresponding author. Departamento de Química e Bioquímica, FCT, Universidade do Algarve, 8005-139 Faro, Portugal. Tel.: +351 289800900; fax: +351289818403.

E-mail address: ttiago@ualg.pt (T. Tiago).

1 These authors contributed equally to this work.
}

entrance/exit to the active site at the rear opening of the Pi-tube opposite to the "front door". This "back-door" appears to be both a likely approach route for a water molecule to attack the $\gamma$ phosphate during ATP hydrolysis, and a likely exit route of the cleaved $\gamma$-phosphate after hydrolysis $[10,11]$. The $\gamma$-phosphate of ATP is partially visible in the structure of S1dC complexed with MgADP.BeFx or MgATP, in which there is a clear opening of the Pi-tube into the large cleft that divides the $50 \mathrm{kDa}$ fragment into upper and lower domains. In contrast, in the $\mathrm{S} 1 \mathrm{dC}$ structures complexed with $\mathrm{MgADP} \cdot \mathrm{VO}_{4}$ or $\mathrm{MgADP} \cdot \mathrm{AlF}_{4}$ the opening of the Pi-tube is blocked off by a closure of the $50 \mathrm{kDa}$ cleft and the $\gamma$-phosphate is no longer visible (Fig. 1). The latter conformational change has been suggested to be necessary for the hydrolysis of ATP [12-14] meaning that the phosphate group will be trapped in the Pi-tube until a second conformational rearrangement occurs, which was proposed to occur upon myosin rebinding to actin [4]. Besides elucidating the kinetics of ATP hydrolysis, this "back door" mechanism has been used to explain a number of experimental observations, including the 

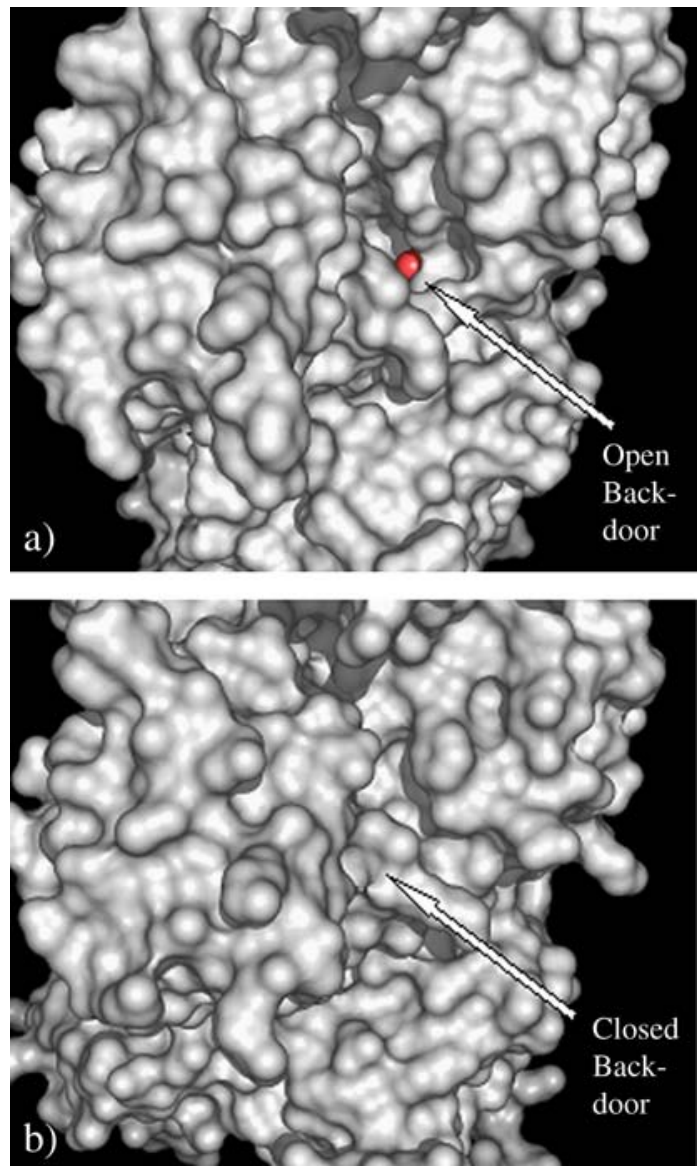

Fig. 1. Back-door view of the structure of the S1 domain of Dictyostelium discoideum myosin in complex with ATP (a) and $\mathrm{MgADP} \cdot \mathrm{VO}_{4}$ (b). The ATP $\gamma$-phosphate group oxygen (in red) can be seen through the open back-door in the S1.MgATP complex. Structures were obtained from the PDB; accession numbers are given in Methods.

formation of stable myosin $\cdot \mathrm{MgADP} \cdot$ vanadate complexes in muscle fibres [10].

The mechanism of inhibition of the myosin ATPase activity by vanadate is relatively well characterized. Monomeric vanadate $\left(\mathrm{VO}_{4}^{3-}\right)$ mimics the transition state for the $\gamma$-phosphate hydrolysis freezing myosin in a pre-power stroke state [15]. However, there is a considerably difference in the ability of vanadate to inhibit the myosin and the actomyosin ATPase activity. While in the absence of actin vanadate inhibits myosin ATPase activity in the submicromolar concentration range, in the presence of actin much higher concentrations $(>900 \mu \mathrm{M})$ are needed [16,17]. Clearly, the need for such high concentrations strongly suggested that this effect could be due to oligomeric vanadate species that are favored at higher vanadate concentrations $[18,19]$ and likely to occur through a different mechanism. In fact it was recently shown that the ATPase activity of the actomyosin complex is inhibited $(\mathrm{Ki}=0.27 \pm$ $0.05 \mu \mathrm{M})$ by decameric but not by the monomeric form of vanadate $[20,21]$. The results were consistent with binding of decavanadate $\left(\mathrm{V}_{10}\right)$ to the conserved regions of the phosphate binding-loop (P-loop) which shape part of the Pi-tube [22]. This $\mathrm{V}_{10}$ high-affinity binding site produces non-competitive inhibi- tion of the actin-stimulated S1-ATPase activity, without causing dissociation of the ATP-free rigor acto-S1 complex. Moreover, the affinity of $\mathrm{S} 1$ for $\mathrm{V}_{10}$ is modulated by the conformational changes that takes place in S1 during the catalytic cycle, as indicated by the two- to three-fold increase of the dissociation constant produced in the presence of $\mathrm{ADP} \cdot \mathrm{VO}_{4}$ and $\mathrm{ADP} \cdot \mathrm{AlF}_{4}$ [21]. Two questions arose from this work: (1) how does $\mathrm{V}_{10}$ access the P-loop? (2) How can the $\mathrm{V}_{10}$ binding mode account for non-competitive inhibition of the ATPase activity of the actomyosin complex towards the nucleotide and actin?

In order to address the previous questions, we performed a theoretical study in which decavanadate was docked in three different myosin motor domain conformations representing different intermediate states of the contractile cycle. The source for these conformations were three X-ray structures of the $D$. discoideum myosin $\mathrm{S} 1$ fragment, namely the apo (ligand-free) form, and the S1dC $\cdot \mathrm{MgATP}$ and $\mathrm{S} 1 \mathrm{dC} \cdot \mathrm{MgADP} \cdot \mathrm{VO}_{4}$ complexes $[5,8]$. The first two conformations considered (free and ATP-bound) are quite similar and they both show an open back-door conformation characteristic of the pre-hydrolysis state. By binding at this site $\mathrm{V}_{10}$ could block the Pi-tube and prevent ATP hydrolysis without competing with ATP or actin for association with myosin. The third conformation is an analogue of the transition state for ATP hydrolysis, and is expected to bind $V_{10}$ with lower affinity since the back-door is in a closed conformation, rendering the P-loop inaccessible to this ligand.

\section{Methods}

\subsection{Selection and preparation of structures}

Three D. discoideum myosin S1 structures were retrieved from the Brookhaven Protein Databank: ligand-free (PDB code: 1FMV), in complex with MgATP (PDB code: 1 FMW) and in complex with $\mathrm{MgADP} \cdot \mathrm{VO}_{4}(\mathrm{PDB}$ code: $1 \mathrm{VOM}$ ) $[5,8]$. The program Needle (EMBL-EBI site) was used to calculate the percentage identity between the amino acid sequence of myosin II from $D$. discoideum and from rabbit skeletal muscle using a global alignment procedure. Several short loops, missing from the $\mathrm{x}$-ray structures, were built and optimized with the MODELLER software, following standard procedures [23]. All models were then validated with the PROCHECK software [24]. All crystallographic water molecules were removed prior to the docking procedure.

\subsection{Decavanadate and protein parameters}

Coordinates for the decavanadate ion $\left(\mathrm{V}_{10} \mathrm{O}_{28}^{6-}\right)$ were retrieved from the Cambridge Structural Database. There are three non-equivalent positions for vanadium atoms in decavanadate: two VA atoms lie at the center of the equatorial plane, four $\mathrm{VB}$ atoms are in the equatorial plane and four $\mathrm{VC}$ atoms lie in an axial position. Partial charges for all atoms were assigned after SCF calculations of Kempf et al. [25]. These authors found that the inner vanadate oxygens should be close to ionic state, with a charge of -2 , while all other oxygens would have charges close to -1 . The remaining charge should be evenly distributed among the 10 vanadium atoms. For the purpose of this work, a charge of -2 was assigned to the $\mathrm{OA}$ and $\mathrm{OB}$ oxygen, a charge of -1 to all remaining oxygen atoms, and a charge of +2.4 to the 10 vanadium atoms. The non-bond parameters for vanadium were copied from the corresponding iron values in the AutoDock library, a reasonable assumption given the similarity between these two elements. Partial charges for the protein, MgATP and $\mathrm{MgADP}$ atoms were taken from the Amber 95 forcefield library [26]. For the $\mathrm{VO}_{4}$ ion, the total charge of -3 was evenly distributed among the four oxygen atoms. 


\subsection{Molecular docking}

All docking simulations were produced with the software AutoDock 3.0 [27], using Simulated Annealing algorithm (SA) and a total of 128 runs per docked complex. Since no torsional degrees of freedom were assigned to decavanadate, the SA docking algorithm was chosen because it performs better in rigid-body docking situations. For the protein, the lack of conformational flexibility is implied in the algorithm implemented in this version of the AutoDock program.

The termination criterion for docking runs was either a maximum number of 25,0000 energy evaluations or a maximum of 27,000 generations. The docking trajectories were calculated within a cubic grid of size $36 \AA$ and spacing $0.3 \AA$ centered on the active site and the final solutions were clustered with a tolerance of $1.0 \AA$ RMSD (root mean square deviation). Within each cluster, solutions are ranked according to docking energies from lowest to highest.

\section{Results}

The docking of decavanadate was performed on the structure of truncated $D$. discoideum myosin motor domain (S1dC) because the crystallographic structure of rabbit skeletal myosin has not been experimentally solved. Moreover, S1dC offers a greater number of nucleotide-bound complexes with high resolution compared to other myosins. At $43 \%$ sequence identity, the two molecules have a sufficient degree of structural similarity [28] to allow interpretation of the results here presented on the basis of our previously published experimental data on rabbit myosin.

The three myosin $D$. discoideum structures used in the present work were chosen as they represent three distinct states of the actomyosin ATP hydrolysis cycle relevant to the interpretation of our previously published data on the interaction between rabbit skeletal myosin $\mathrm{S} 1$ and decavanadate $[21,22]$. The docking region is limited to a cube with side $36 \AA$ centered on the active site, since $V_{10}$ is experimentally predicted to bind in the vicinity of the catalytic center through the phosphate-loop [22]. Docking calculations with the AutoDock software were run on the three crystal structures, and the results were evaluated in terms of the grouping (clustering) of solutions found in each case. The number of solutions found in each location (back- or front-door) and the lowest docking energy are indicative of the stability of the complex and are displayed in Table 1.

Table 1

Molecular simulation results for decavanadate docking on the surface of the nucleotide-free (Apo), $\mathrm{MgATP}$ and $\mathrm{MgADP} \cdot \mathrm{VO}_{4}$ structures of the $\mathrm{S} 1$ domain of Dictyostelium discoideum myosin

\begin{tabular}{lllll}
\hline & $\begin{array}{l}\text { No. of } \\
\text { solutions at } \\
\text { "Back-door" }\end{array}$ & $\begin{array}{l}\text { No. of } \\
\text { solutions at } \\
\text { "Front-door" }\end{array}$ & $\begin{array}{l}\text { No. of } \\
\text { solutions in } \\
\text { top cluster* }\end{array}$ & $\begin{array}{l}\text { Lowest } \\
\text { energy } \\
\left(\text { Kcal mol }^{-1}\right)\end{array}$ \\
\hline $\begin{array}{c}\text { Apo (1FMV) } \\
\text { MgATP }\end{array}$ & 89 & 2 & 48 & -3.20 \\
$\begin{array}{c}\text { (1FMW) } \\
\text { MgADP·Vi } \\
(1 \mathrm{VOM})\end{array}$ & 0 & 3 & 38 & -2.02 \\
\hline
\end{tabular}

Protein coordinates were taken from the Brookhaven Protein Databank; accession numbers are given in parenthesis. ${ }^{*}$ Cluster with lower docking energies.
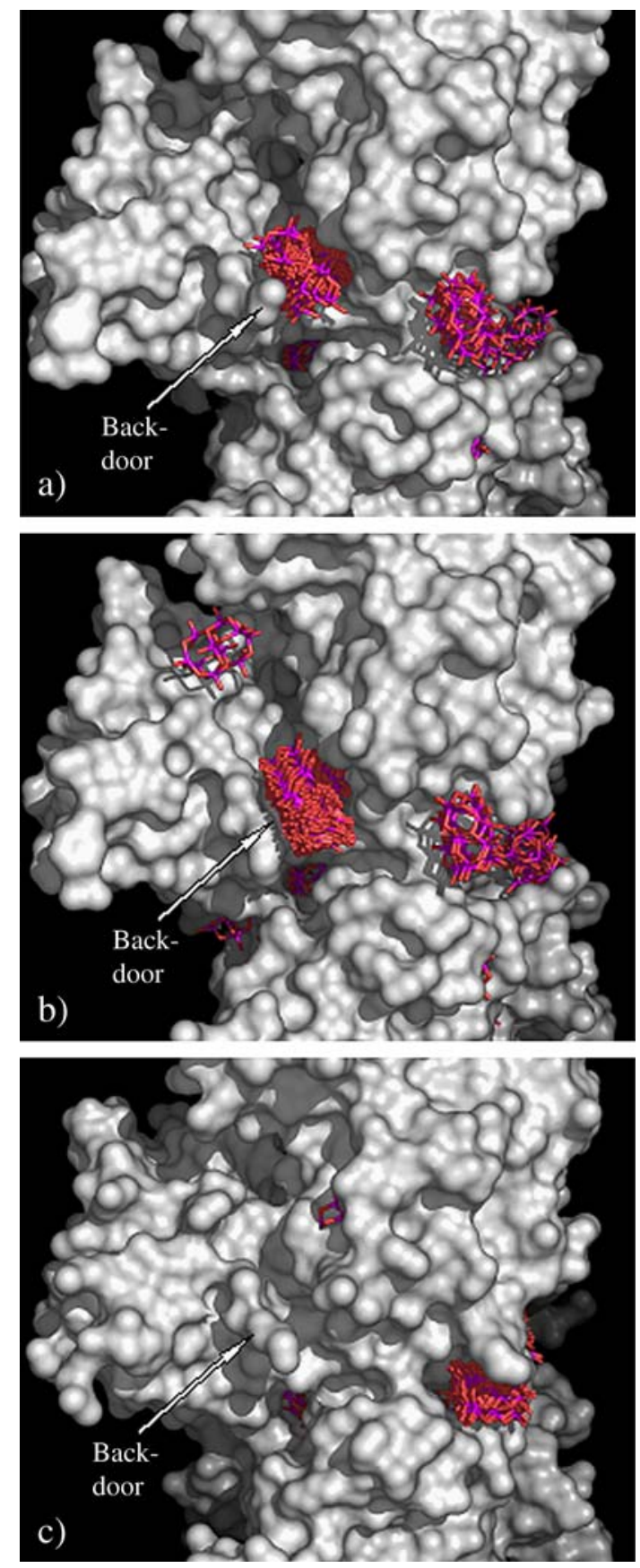

Fig. 2. Resulting docked conformations of decavanadate (represented as sticks) on the surface of the nucleotide-free (a), $\mathrm{Mg} \cdot \mathrm{ATP}$ (b) and $\mathrm{Mg} \cdot \mathrm{ADP}^{-\mathrm{VO}_{4}}$ (c) structures of the S1 domain of Dictyostelium discoideum myosin. Docking was performed as described in Methods. The back-door entrance and the nucleotide binding site are on the left and right side of each structure, respectively.

\subsection{The apo-myosin structure}

The crystal structure of S1dC myosin in its ligand-free (apo) form is believed to be representative of the pre-hydrolysis state after dissociation from actin [8]. The back-door is open in this structural state (Fig. 1a), allowing $\mathrm{V}_{10}$ to easily access the Ploop from this side. Of the 128 docking runs calculated for this structure, 89 ended with $\mathrm{V}_{10}$ docked on the back-door (Table 1 and Fig. 2a) and 79 of these conformations were the lowest 
energy conformations, when compared to the remaining 49 runs. The lowest docking energy (in the back-door) was -3.20 $\mathrm{kcal} \mathrm{mol}^{-1}$ (this energy value is only indicative, due to the highly idiosyncratic nature of $\mathrm{V}_{10}$ when compared to the test set used to parameterize Autodock). The preference thus shown by $\mathrm{V}_{10}$ to bind at the back-door region leads support to our previous finding that $\mathrm{V}_{10}$ does not compete with ATP for binding to myosin $[21,22]$. Also, this location is consistent with the non-competitive behavior of $\mathrm{V}_{10}$ towards actin binding to myosin as it does not interfere with the actin binding interface.

A careful screening of the back-door solutions reveals another feature correlated to our previous experimental findings: back-door binding of $\mathrm{V}_{10}$ brings it very close to Ser181 (Fig. 3a), a residue located on the 178-185 sequence which forms the phosphate binding-loop (P-loop) motive, previously shown to be cleaved, upon irradiation, in the presence of $\mathrm{V}_{10}$ [22]. In the "front-door", besides being farther from Ser-181 $(11.84 \AA)$ than in the back-door $(6.27 \AA$ for the lowest docked energy conformation), the docked location of decavanadate is partially shielded by side chains of other residues which would prevent the access to Ser-181. One must note however that the use of a rigid protein model in the docking procedure makes these distances merely indicative.

\subsection{The MgATP-myosin complex}

The crystal structure of S1dC myosin complexed with ATP is also an open back-door structure, very similar to the apo-S1dC structure already discussed above. As pointed out by Bauer et al. [8], this could explain the high affinity of myosin for ATP, and is in agreement with this structure belonging to a pre-hydrolysis state, and thus unable to hydrolyze ATP. Given the extent of structural similarity, it is no surprise that the docking results are very similar to those obtained with the apo-S1dC structure. Of the 128 docking runs, 95 ended with $\mathrm{V}_{10}$ docked in the back- door region, and 62 of theses conformations were the solutions with lower energy (Table 1 and Fig. 2b). The lowest energy for a docked conformation was slightly higher than in the apo-S1dC docking $(-2.02 \mathrm{kcal} / \mathrm{mol})$, as expected because of the repulsion between the negative charge of $V_{10}$ and ATP (however, see the previous remark on the significance of Autodock energies). The preference for $\mathrm{V}_{10}$ binding at the back-door is again clear, in spite of the electrostatic effect of ATP at the active site. This result agrees well with the experimentally observed noncompetitive effect of $\mathrm{V}_{10}$ towards myosin ATP hydrolysis [21]. The most stable conformation of $V_{10}$ is again close to Ser181, but not as close as in the apo-S1dC dockings (Fig. 3b). This increased distance (approximately $2 \AA$ for the lowest energy conformations) could explain why only partial $V_{10}$ photocleavage of myosin is attained in the presence of nucleotide [22].

\subsection{The $\mathrm{MgADP} \cdot \mathrm{VO}_{4}-$ myosin complex}

The $\mathrm{S} 1 \mathrm{dC}-\mathrm{MgADP}-\mathrm{VO}_{4}$ crystal structure is believed to be representative of the post-hydrolysis transient state before the power-stroke $[5,29,30]$. In this state, the $50-\mathrm{kDa}$ cleft has closed (Fig. 1b), blocking the Pi-tube and preventing both the release of $\mathrm{Pi}$ and the access of $\mathrm{V}_{10}$ to the back-door and consequently the P-loop. Accordingly, out of the 128 simulation runs there were 79 final $V_{10}$ conformations docked at the entrance of the ATP-binding site ("front-door"), and these were the lowest energy solutions (Table 1 and Fig. 2c). With the access to the P-loop barred, binding at the back-door region no longer represents an energetically favorable solution, and the front door remains as the only favorable solution for docking $\mathrm{V}_{10}$ on the myosin surface. The smaller stabilization offered by binding at the nucleotide site could explain the experimentally observed two- to three-fold increase for the dissociation constant of the $\mathrm{V}_{10}$-myosin complex in the presence of $\mathrm{ADP} \cdot \mathrm{VO}_{4}$ or $\mathrm{ADP} \cdot \mathrm{AlF}_{4}$ (another analogue of the transition
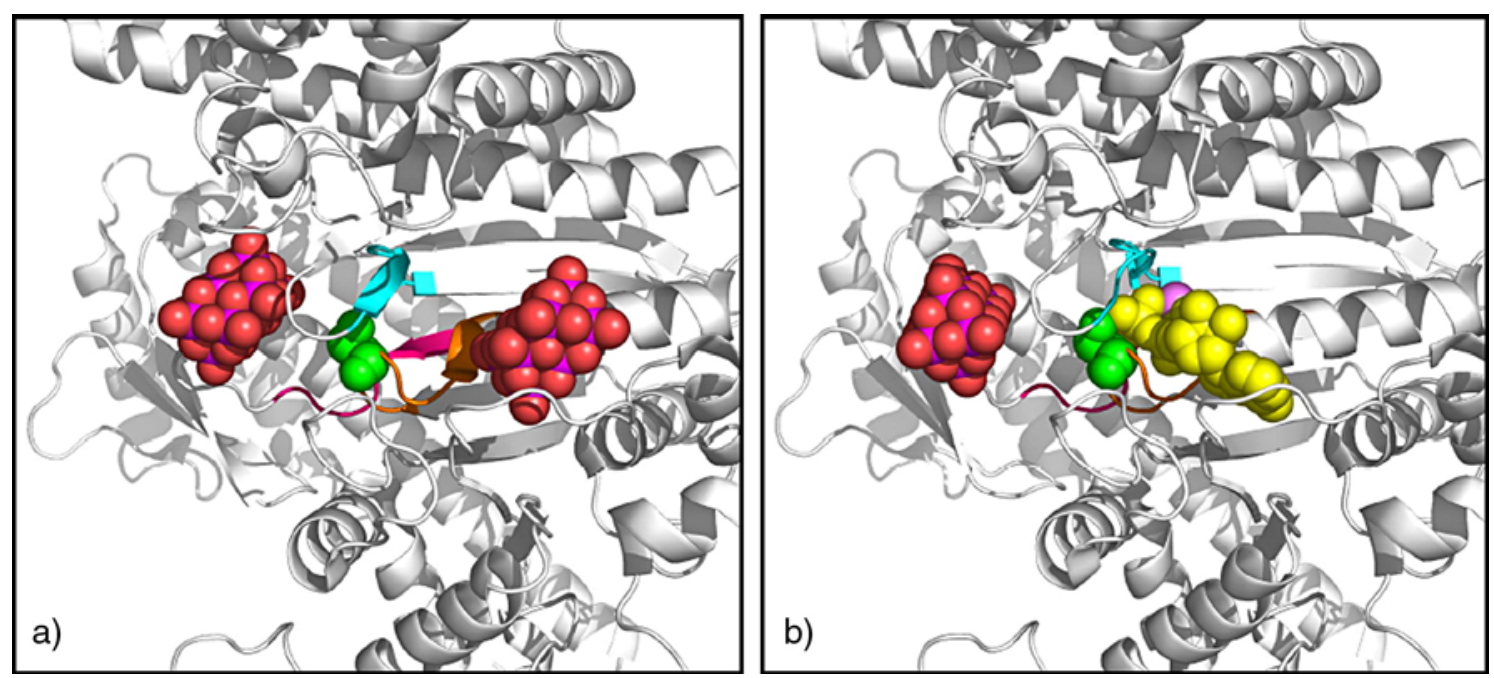

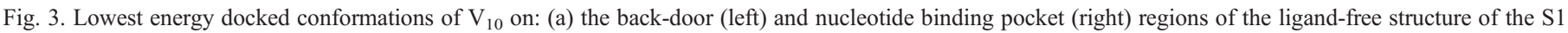

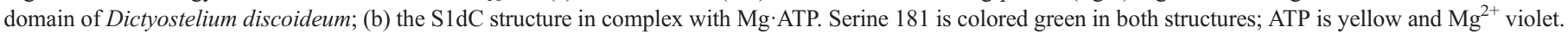

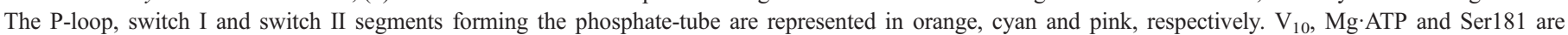
represented as spheres. 
state for ATP hydrolysis; $\mathrm{S} 1 \mathrm{dC} \cdot \mathrm{ADPAlF}_{4}$ structure is very similar to the $\mathrm{ADP} \cdot \mathrm{VO}_{4}$ structure) [21]. Therefore, if different conformational states of myosin display different affinities for $\mathrm{V}_{10}$, it is expected that an altered distribution of conformational states during the steady state catalytic activity takes place in the presence of $\mathrm{V}_{10}$.

\section{Discussion}

The computational molecular docking calculations presented here, together with previous experimental data, lend strong support for the binding of $\mathrm{V}_{10}$ at the back-door of the $\mathrm{S} 1 \mathrm{dC}$ myosin fragment in the free or pre-hydrolysis nucleotide-bound state. This binding location both explains how the $10 \AA$ decavanadate molecule gains access to the P-loop without having to squeeze through the narrow entrance of the phosphate-tube at the nucleotide binding site, and provides a simple mechanism for the experimentally observed noncompetitive pattern of inhibition of $\mathrm{V}_{10}$ towards binding of both ATP and actin to myosin. By binding at the back-door, at the end of the Pi-tube, $V_{10}$ will not interfere with the nucleotide binding site or the actin binding surface. Photo-cleavage of myosin by $\mathrm{V}_{10}$ at the Ser-181 position can also be accounted for, since the lowest energy docked solutions of $V_{10}$ at the backdoor place it in very close contact with this residue. Binding of $\mathrm{V}_{10}$ to the back-door is also supported by the experimentally observed strong quenching of IAEDANS fluorescence by $\mathrm{V}_{10}$ [21], when the probe is bond to a cysteine residue close to the back-door (Cys697). Also consistent with this picture is the previously observed competition of $\mathrm{V} 10$ binding with $\mathrm{AP}_{5} \mathrm{~A}$ [21], a $\gamma$-phosphate modified nucleotide analogue that has been shown to bind myosin in a bimodal way that extends from the catalytic crevice through the Pi-tube and to the $50 \mathrm{kDa}$ cleft leading to the back-door [31]. However, the question still remains: at which step in the actomyosin ATPase cycle does $V_{10}$ bind to myosin?

Since the closed states of myosin display low affinity for $\mathrm{V}_{10}$, binding must take place when the back-door is open, either at the pre-hydrolysis or the "power-stroke" states of the actomyosin ATP hydrolysis cycle. In the latter state myosin is strongly bound to actin, so $\mathrm{V}_{10}$ would have to interfere with formation of the transient acto-myosin complex. However, we have previously shown by light-scattering studies that $\mathrm{V}_{10}$ binding does not dissociate the ATP-free rigor acto-myosin complex [21]. On the other hand, these experimental results indicated a positive correlation between the time after addition of ATP needed for the re-association of actin to myosin in the presence of $\mathrm{V}_{10}$ and the extent of $\mathrm{V}_{10}$ inhibition of the actomyosin ATPase activity. Besides, decavanadate addition with MgADP is without effect on the actomyosin complex. Together, these results show that while $\mathrm{V}_{10}$ does not promote dissociation of the acto-myosin complex, it does prevent its formation until ATP level in the solution is sufficiently low for the re-association process to occur. Consequently, $\mathrm{V}_{10}$ must bind to myosin at its dissociated state, most likely the pre-hydrolysis state after actin release and before closure of the $50 \mathrm{kDa}$ cleft and concomitantly a)
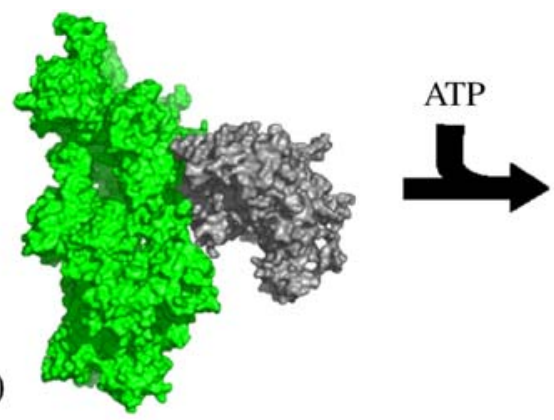

d)
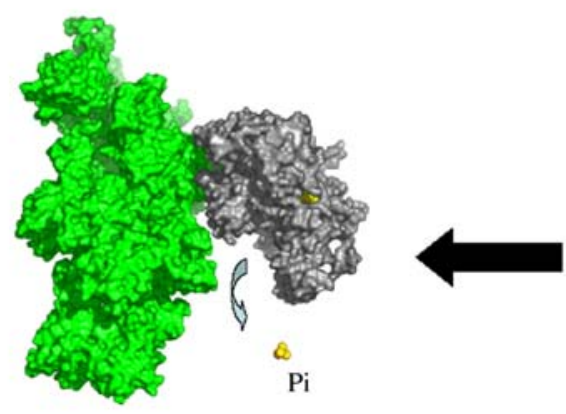

c)

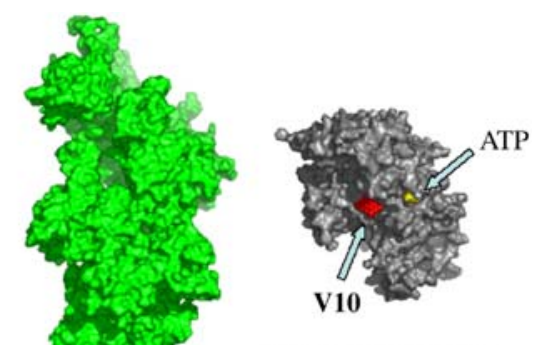

b)

Open back-door

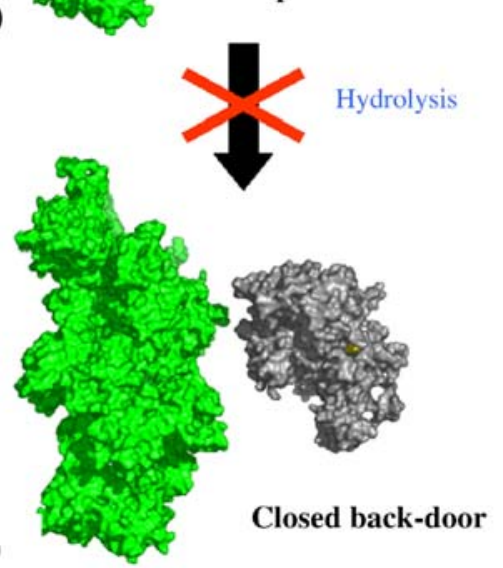

Fig. 4. Relevant steps of the actomyosin ATP hydrolysis cycle schematically represented as perturbed by $\mathrm{V}_{10}$ binding: ATP binding to myosin in the rigor state (a) promotes opening of the 50-kDa cleft leading to dissociation of the actomyosin complex (b). In the absence of $\mathrm{V}_{10}$, closure of the active site leads to ATP hydrolysis and a conformational change of the S1 domain (c). At this point, the back-door is closed and the hydrolyzed Pi group is trapped inside the active site until myosin rebinds actin, leading to the opening of the back-door and Pi release (d). When present, $\mathrm{V}_{10}$ acts as "back-door stop", blocking closure of the 50-kDa cleft and concomitant closure of the back-door thus preventing ATP hydrolysis. 
the back-door. Decavanadate binding at this step would act as a "back-door stop" blocking the conformational change necessary to carry out the ATP- $\gamma$-phosphate hydrolysis. This proposal is schematically represented in Fig. 4. When attempting to extrapolate the myosin behavior in muscle fibers from the above proposal, one must keep in mind that all structures used in this work are static intermediates, frozen snapshots taken out of the conformational complexity of myosin in solution. As such, there may be other transient states, as of yet unknown, playing a key role in the myosin cycle in vivo.

Very often studies describing interactions of vanadate with proteins consider only monomeric vanadate as the active species, disregarding the possible contribution of other vanadate oligomers which are favored at higher vanadate concentrations $[18,19]$. Unlike the simple and labile vanadate oligomers including monomeric (V1), dimeric (V2), tetrameric (V4) and pentameric (V5) species, which interchange with each other on the millisecond to second time scale in neutral and alkaline aqueous solutions, decameric species (V10) interconversion with the other vanadate oligomers occurs on a considerably longer time scale [32]. Although above $\mathrm{pH} 6$ decavanadate is not the most thermodynamically stable species, it can persist for limited time periods because hydrolysis into other vanadate oligomers is very slow which makes it an excellent molecular probe to investigate the nature of polyoxoanions interactions with proteins. Among the free or complexed forms of vanadate, decameric species is one of the most potent inhibitors. The enzymes inhibited by decavanadate appear to be all prearranged to bind phosphoryl groups, but V10 is structurally different from V1 and cannot mimic orthophosphate in its interaction with enzymes. Moreover, decavanadate inhibition is by no means a general property of all the enzymes that bind phosphoryl groups and therefore the structural and conformational characteristics of the enzymes must be important. For example, Pezza et al. [33] have shown that the Walker A motive of ABC ATPases is a highly adapted anion-binding domain that can bind decavanadate with high affinity. Unlike V1, decavanadate interacts outside the Walker A loop (corresponding to the P-loop in myosin) and is stabilized by certain residues nearby. Therefore, according to these authors, if the presence of these residues is essential for the binding stabilization, only some members of the ABC superfamily are able to interact with decavanadate.

In what concerns myosin, vanadate is able to populate different conformational states of the myosin ATPase cycle depending on its oligomerization state. While monomeric vanadate $\left(\mathrm{VO}_{4}^{3-}\right)$ mimics the transition state for the $\gamma$-phosphate hydrolysis blocking myosin in a pre-power-stroke state, decameric vanadate induces the formation of the intermediate myosin $\cdot M g A T P \cdot V_{10}$ complex blocking the actomyosin cycle presumably in the pre-hydrolysis state. Therefore, the mode of action of vanadate on the inhibition of the actin-stimulated myosin ATPase activity depends, at least in part, on the charge and size of the vanadate species, being favored for those with higher oligomerization state, such as the decameric species. The specific phosphate-binding domains in the vicinity of the backdoor provide electrostatic interactions which favor an approach of this polyanionic species to the rear opening of the Pi-tube. In addition, the large size of decavanadate is likely to interfere with movements associated with closure of the $50-\mathrm{kDa}$ cleft and concomitant closure of the back-door. This allows rationalizing on simple grounds (1) the non-competitive inhibition of $\mathrm{V}_{10}$ towards both actin and ATP of the actin-stimulated myosinATPase activity; (2) the delayed re-association of the actomyosin complex but without a dissociation of the ATP-free rigor complex in the presence of decavanadate; (3) the preference of $\mathrm{V}_{10}$ to bind at the back-door entrance of the Pi-tube, but only on the "open" structures were there is access to the phosphate binding-loop. Finally, this study lends further support to the myosin back-door mechanism hypothesis and spurs the use of decavanadate as a biochemical tool to gain a deeper knowledge of the myosin back-door modulation and the relevance of its conformational changes in the process of muscle contraction.

\section{Acknowledgments}

This work has been supported by Joint Spanish-Portuguese Grant HP2004-0080 (to C.G.-M. and M.A.), by POCTI program financed through FEDER for the research project 38191/QUI/2001 (to M.A.), and by Grant 3PR05A078 of the Junta de Extremadura (to C.G.-M.). Dr. T. Tiago is the recipient of a post-doctoral fellowship (SFRH/BPD/20777/ 2004) from the Portuguese Foundation for Science and Technology (FCT).

\section{References}

[1] Y.Y. Toyoshima, S.J. Kron, E.M. McNally, K.R. Niebling, C. Toyoshima, J.A. Spudich, Myosin subfragment-1 is sufficient to move actin filaments in vitro, Nature 328 (1987) 536-539.

[2] J.E. Walker, M. Saraste, M.J. Runswick, N.J. Gay, Distantly related sequences in the alpha- and beta-subunits of ATP synthase, myosin, kinases and other ATP-requiring enzymes and a common nucleotide binding fold, EMBO J. 1 (1982) 945-951.

[3] I. Rayment, W.R. Rypniewski, K. Schmidt-Base, R. Smith, D.R. Tomchick, M.M. Benning, D.A. Winkelmann, G. Wesenberg, H.M. Holden, Three-dimensional structure of myosin subfragment-1: a molecular motor, Science 261 (1993) 50-58.

[4] A.J. Fisher, C.A. Smith, J. Thoden, R. Smith, K. Sutoh, H.M. Holden, I. Rayment, Structural studies of myosin:nucleotide complexes: a revised model for the molecular basis of muscle contraction, Biophys. J. 68 (1995) 19S-26S (discussion 27S-28S).

[5] C.A. Smith, I. Rayment, X-ray structure of the magnesium(II).ADP. vanadate complex of the Dictyostelium discoideum myosin motor domain to 1.9 A resolution, Biochemistry 35 (1996) 5404-5417.

[6] C.B. Bauer, P.A. Kuhlman, C.R. Bagshaw, I. Rayment, X-ray crystal structure and solution fluorescence characterization of $\mathrm{Mg} .2^{\prime}\left(3^{\prime}\right)-\mathrm{O}-(\mathrm{N}-$ methylanthraniloyl) nucleotides bound to the Dictyostelium discoideum myosin motor domain, J. Mol. Biol. 274 (1997) 394-407.

[7] A.M. Gulick, C.B. Bauer, J.B. Thoden, I. Rayment, X-ray structures of the MgADP, MgATPgammaS, and MgAMPPNP complexes of the Dictyostelium discoideum myosin motor domain, Biochemistry 36 (1997) $11619-11628$

[8] C.B. Bauer, H.M. Holden, J.B. Thoden, R. Smith, I. Rayment, X-ray structures of the apo and MgATP-bound states of Dictyostelium discoideum myosin motor domain, J. Biol. Chem. 275 (2000) 38494-38499.

[9] A.M. Gulick, C.B. Bauer, J.B. Thoden, E. Pate, R.G. Yount, I. Rayment, X-ray structures of the Dictyostelium discoideum myosin motor domain with six non-nucleotide analogs, J. Biol. Chem. 275 (2000) 398-408. 
[10] R.G. Yount, D. Lawson, I. Rayment, Is myosin a "back door" enzyme? Biophys. J. 68 (1995) 44S-47S (discussion 47S-49S).

[11] J.D. Lawson, E. Pate, I. Rayment, R.G. Yount, Molecular dynamics analysis of structural factors influencing back door pi release in myosin, Biophys. J. 86 (2004) 3794-3803.

[12] N. Sasaki, K. Sutoh, Structure-mutation analysis of the ATPase site of Dictyostelium discoideum myosin II, Adv. Biophys. 35 (1998) 1-24.

[13] M. Furch, S. Fujita-Becker, M.A. Geeves, K.C. Holmes, D.J. Manstein, Role of the salt-bridge between switch-1 and switch-2 of Dictyostelium myosin, J. Mol. Biol. 290 (1999) 797-809.

[14] N. Okimoto, K. Yamanaka, J. Ueno, M. Hata, T. Hoshino, M. Tsuda, Theoretical studies of the ATP hydrolysis mechanism of myosin, Biophys. J. 81 (2001) 2786-2794.

[15] C.C. Goodno, Inhibition of myosin ATPase by vanadate ion, Proc. Natl. Acad. Sci. U. S. A. 76 (1979) 2620-2624.

[16] C.C. Goodno, E.W. Taylor, Inhibition of actomyosin ATPase by vanadate, Proc. Natl. Acad. Sci. U. S. A. 79 (1982) 21-25.

[17] S.J. Smith, E. Eisenberg, A comparison of the effect of vanadate on the binding of myosin-subfragment-1.ADP to actin and on actomyosin subfragment 1 ATPase activity, Eur. J. Biochem. 193 (1990) 69-73.

[18] D.C. Crans, Aqueous chemistry of labile oxovanadates: relevance to biological studies, Comments Inorg. Chem. 16 (1994) 1-33.

[19] D.C. Crans, Enzyme interactions with labile oxovanadates and other polyoxometalates, Comments Inorg. Chem. 16 (1994) 35-76.

[20] M. Aureliano, Vanadate oligomer interactions with myosin, J. Inorg. Biochem. 80 (2000) 141-143.

[21] T. Tiago, M. Aureliano, C. Gutierrez-Merino, Decavanadate binding to a high affinity site near the myosin catalytic centre inhibits F-actinstimulated myosin ATPase activity, Biochemistry 43 (2004) 5551-5561.

[22] T. Tiago, M. Aureliano, J.J. Moura, Decavanadate as a biochemical tool in the elucidation of muscle contraction regulation, J. Inorg. Biochem. 98 (2004) 1902-1910.

[23] A. Sali, T.L. Blundell, Comparative protein modelling by satisfaction of spatial restraints, J. Mol. Biol. 234 (1993) 779-815.

[24] R.A. Laskowski, M.W. Macarthur, D.S. Moss, J.M. Thornton,
Procheck-A program to check the stereochemical quality of protein structures, J. Appl. Crystallogr. 26 (1993) 283-291.

[25] J.Y. Kempf, M.M. Rohmer, J.M. Poblet, C. Bo, M. Benard, Relative basicities of the oxygen sites in [V10o28]6--An analysis of the abinitio determined distributions of the electrostatic potential and of the laplacian of charge-density, J. Am. Chem. Soc. 114 (1992) 1136-1146.

[26] W.D. Cornell, P. Cieplak, C.I. Bayly, I.R. Gould, K.M. Merz, D.M Ferguson, D.C. Spellmeyer, T. Fox, J.W. Caldwell, P.A. Kollman, A 2nd generation force-field for the simulation of proteins, nucleic-acids, and organic-molecules, J. Am. Chem. Soc. 117 (1995) 5179-5197.

[27] G.M. Morris, D.S. Goodsell, R.S. Halliday, R. Huey, W.E. Hart, R.K. Belew, A.J. Olson, Automated docking using a Lamarckian genetic algorithm and an empirical binding free energy function, J. Comput. Chem. 19 (1998) 1639-1662.

[28] C. Sander, R. Schneider, Database of homology-derived protein structures and the structural meaning of sequence alignment, Proteins 9 (1991) 56-68.

[29] A.J. Fisher, C.A. Smith, J.B. Thoden, R. Smith, K. Sutoh, H.M. Holden, I. Rayment, X-ray structures of the myosin motor domain of Dictyostelium discoideum complexed with MgADP.BeFx and MgADP.AlF4, Biochemistry 34 (1995) 8960-8972.

[30] R. Dominguez, Y. Freyzon, K.M. Trybus, C. Cohen, Crystal structure of a vertebrate smooth muscle myosin motor domain and its complex with the essential light chain: visualization of the pre-power stroke state, Cell 94 (1998) 559-571.

[31] E. Pate, N. Naber, M. Matuska, K. Franks-Skiba, R. Cooke, Opening of the myosin nucleotide triphosphate binding domain during the ATPase cycle, Biochemistry 36 (1997) 12155-12166.

[32] D.C. Crans, C.D. Rithner, L.A. Theisen, Application of time-resolved V-51 2d NMR for quantitation of kinetic exchange pathways between vanadate monomer, dimer, tetramer, and pentamer, J. Am. Chem. Soc. 112 (1990) 2901-2908.

[33] R.J. Pezza, M.A. Villarreal, G.G. Montich, C.E. Argarana, Vanadate inhibits the ATPase activity and DNA binding capability of bacterial MutS. A structural model for the vanadate-MutS interaction at the Walker A motif, Nucleic Acids Res. 30 (2002) 4700-4708. 